\title{
Review article: radiofrequency neurotomy for the treatment of sacroiliac joint syndrome
}

\author{
Stasia Bochnowski Muhlner
}

Published online: 31 March 2009

(c) The Author(s) 2009. This article is published with open access at Springerlink.com

\begin{abstract}
We review here the latest studies on efficacy of and techniques employed in radiofrequency neurotomy of the sacroiliac joint. Radiofrequency neurotomy has been shown to have limited evidence in treating sacroiliac joint syndrome. Variability in results has been attributed to individual nerve patterns, improper diagnosis, and selection of patients, as well as variability in the procedure itself. Future studies will be needed to focus on the type of radiofrequency neurotomy and positioning of electrodes to better evaluate efficacy.
\end{abstract}

Keywords Sacroiliac joint - Low back pain ·

Radiofrequency neurotomy · Radiofrequency ablation .

Radiofrequency denervation - Radiofrequency nerve block

\section{Introduction}

For the general practitioner, sacroiliac joint syndrome (SIJS) can sometimes be overlooked as a cause of low back pain [1]. However, a recent systematic review by Hansen et al. [2] revealed that the sacroiliac joint (SIJ) is estimated by a double nerve block paradigm to be a source of low back pain in 10-27\% of people with chronic low back pain.

Painful conditions of the SIJ are known to result from infection [3], malignancy [4], pregnancy [5], and either acute or repetitive trauma [5]. Symptoms include low back, buttock, and thigh pain, and sometimes sciatic-like radiating pain. SIJS has been shown to occur bilaterally or unilaterally [6].

S. B. Muhlner $(\square)$

Keck School of Medicine at USC, Los Angeles, CA, USA

e-mail: smuhlner@usc.edu
The review by Hansen et al. [2] concluded that while there are no historical, physical, or radiological features to definitively diagnose SIJ pain, comparative, controlled anesthetic blocks have moderate evidence of validity in diagnosis. Comparative controlled anesthetic blocks used in the studies covered by the review consisted of a $2 \%$ lidocaine injection to screen for SIJ dysfunction, followed by a $0.5 \%$ bupivicaine injection in patients who had a positive response to the lidocaine block, in order to confirm true SIJ dysfunction. A positive response to either block was considered to be relief of pain, though studies differed on the amount and duration of pain relief that qualified as a positive response. The innervation of the SI joint ranges from the posterior primary rami of L-4 through S-3 and the anterior primary rami from L-2 through S-2 [7]. Innervation is variable; therefore, presentations of symptoms are often variable as well. In addition, pain may be referred from dermatomes or adjacent structures, and different sections of the SI joint may be damaged, also accounting for symptom variability [8] (Fig. 1).

\section{Diagnosis}

There are a variety of physical exam tests that have been used to help elucidate whether the SI joint may be a contributing or isolated cause of low back pain. These include the FABER test, distraction test, the compression test, the Gaenslen's test, and the Patrick's test, among others. However, there has been documented poor inter- and intratester reliability with these tests. In addition, Dreyfuss et al. [9] found positive results in up to $20 \%$ of asymptomatic patients, suggesting poor specificity of using such exams for diagnosis. Slipman et al. [10], however, demonstrated a positive predictive value of $60 \%$ in diagnosing SIJS in patients with a minimum of three positive provocative SIJ 


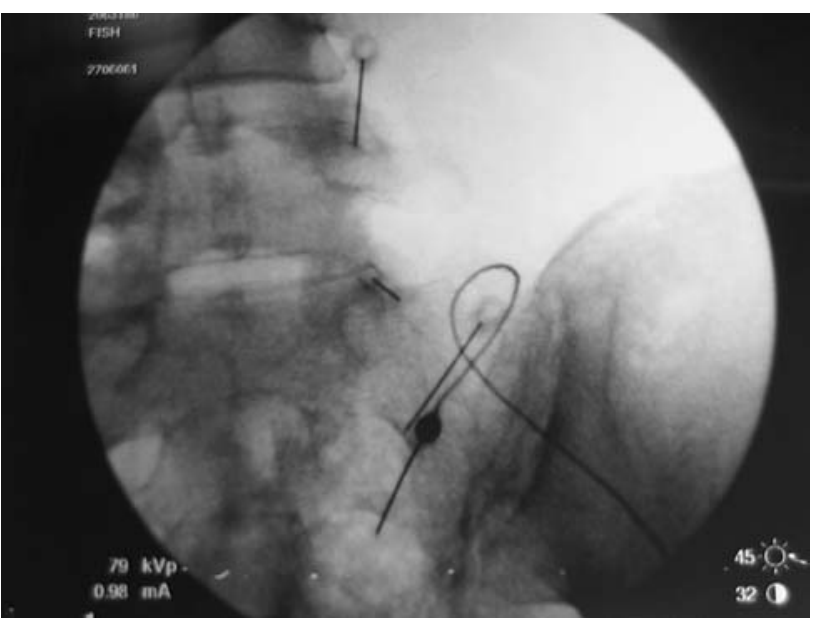

Fig. 1 Radiofrequency ablation of the medial branch nerves to the right SIJ: L5, S1, S2, S3

maneuvers. Lasett et al. [11] confirmed some utility to clinical exam by providing evidence that employing a McKenzie evaluation to exclude discogenic pain and a composite of three or more SIJ pain provocation tests has clinically useful diagnostic accuracy when compared with a reference standard of double block.

While the clinical exam may prove inaccurate, imaging is equally so. Plain films X-rays are rarely diagnostic in SIJ pain syndrome. Degenerative changes of the SIJ seen on Xray have proven clinically insignificant because they are commonly observed in asymptomatic individuals. Similarly, computed tomography (CT), although able to demonstrate previously established bony changes, offers little diagnostic value. A study by Vogler et al. [12] of 45 asymptomatic subjects showed that over $24 \%$ of those over 50 years old showed degenerative SIJ changes on plain film and similar results using CT. Magnetic resonance imaging (MRI) enables visualization of the soft-tissue anatomy of the SIJ and the ability to see septic, inflammatory, or stress-related changes in the bones. However, for typical SIJ pain syndrome, MRI specificity is only 54\% [13]. MRI can, however, be a valuable tool for helping exclude disk herniation, notably for L5-S1 level pathology. Bone scans and single photon emission computed tomography (SPECT) have similarly shown poor sensitivity, at $12.9-65 \%$ and $9.1 \%$, respectively [13-16].

As mentioned previously, the gold standard for the diagnosis of SIJS is diagnostic injection under fluoroscopic guidance [17] using a double injection paradigm: one lidocaine injection to screen for SIJ dysfunction, followed by a bupivicaine injection in patients who had a positive response to the lidocaine block, in order to confirm true SIJ dysfunction [18]. Using two comparative blocks is a more reliable method to establish the diagnosis of intra-articular SIJ pain, as one uncontrolled, single injection has a false positive rate of $20 \%$ [2]. These blocks should target the intra-articular SIJ, not the sacral lateral branches. This is because the sacral lateral branches have fascial planes and ligaments that limit the spread of injected anesthetics [19]. It is equally as important to exclude other sources of the patient's pain, such as the facet joint or discs, through zygapophyseal joint blocks or diskography, respectively [19].

\section{Treatment}

Primary management of acute SI joint dysfunction includes anti-inflammatory medication, heat and ice modalities, and rest. Once the pain has lessened, treatment should involve pelvic stabilization exercises for postural control, as well as muscle balancing of the trunk and lower extremities. Using a sacroiliac belt can provide proprioceptive awareness and give patients more confidence in their daily activities. Procedures that have been used to treat SIJ dysfunction include injection of anesthetic; prolotherapy, which involves injecting a chemical irritant into ligaments to promote collagen proliferation and increase stability; cryotherapy, which involves exposing the lateral nerve branches of the SIJ to liquid or gas nitrogen to promote necrosis of nerves or to create an inflammatory response to increase collagen proliferation and stability; and radiofrequency neurotomy, which involves ablation of the SIJ nerve branches. However, more research into these various approaches is needed to determine efficacy. Finally, surgery can be considered, though its use for SIJ dysfunction is controversial [20].

\section{Radiofrequency neurotomy}

Although radiofrequency neurotomy has been used and has proven efficacy for zygapophyseal (facet) joint pain [19], radiofrequency neurotomy for SIJ pain is only recently emerging as a possibly effective treatment under study. Radiofrequency neurotomy involves using energy in the radiofrequency range to cause necrosis of specific nerves and in so doing, relieve pain. Radiofrequency neurotomy, however, does not come without risks: infection, hematoma formation, burns, and neural damage such as cutaneous dysesthesia or hypoesthesia are all potential adverse outcomes $[2,21]$. Radiofrequency neurotomy can be performed as an outpatient procedure, with the patient able to go home within a few hours, and it can be repeated if after several months the nerves regenerate and pain returns [19]. It is recommended that radiofrequency neurotomy be undertaken only after conservative measures have been exhausted first [20] in a patient with SIJ dysfunction diagnosed by concordant response to injection. 
Nerve variability

Limited success in pain relief for SIJS has been attributed to the variability of the courses of lateral branch nerves, as well as continued debate over the exact innervation of the SIJ [17]. The anterior portion of the SIJ is believed to be innervated by the posterior rami of L1-S2, with possible additional innervation from the obturator nerve, superior gluteal nerve, and lumbosacral trunk, while the posterior portion of the SIJ is believed to be innervated by the posterior rami of L4-S3 [22]. Radiofrequency neurotomy has targeted the L5 dorsal ramus and its branches to the SI joint, as well as the lateral branches of the S1-S3 dorsal rami. Clearly, not all possible nerves that can contribute to SI joint pain are targeted by this procedure [19]. Interestingly, radiofrequency neurotomy targets the dorsal rami and is thus more effective for extra-articular rather than intra-articular joint pain, since there is no known anterior innervation to the dorsal SIJ ligamentous complex [19]. Although radiofrequency neurotomy is performed on dorsal rami, nerve blocks to confirm SIJ pain are not done on dorsal rami because injected anesthetics are limited by fascia and ligaments in that area. Therefore, confirmatory blocks are approximated by injecting intra-articularly $[19,21]$.

In addition, nerves may take circuitous routes in different people, leading to difficulty in targeting the exact location of nerves during the radiofrequency neurotomy procedure. In fact, in a cadaveric study by Yin, the number and location of sacral lateral branch nerves varied markedly, and nerves more often than not took an indirect path to the SI joint complex [21]. Techniques such as sensory stimulation-guided radiofrequency neurotomy have been developed to overcome such individual variability and to protect nearby vessels and other neural structures [21,23]. Sensory-guided radiofrequency neurotomy involves using minimal threshold stimulation voltages to cause a local sensory response, which allows for better identification of symptomatic (pain transmitting) versus asymptomatic (non-pain transmitting) nerves. Yin et al. [21] points out that the negative study by Ferrante did not use sensory stimulation.

Teaching texts have suggested performing radiofrequency lesions at the superior lateral portion of the $\mathrm{S} 2$ and S3 foramina, at the medial branches of the higher dorsal rami in the lumbar region, at the sacral ala and SIJ junction, and along the posterior SIJ long axis. However, Vallejo asserts that there are no evidence-based studies to support such targets [22]. In the positive-outcome studies on radiofrequency neurotomy reviewed by Hansen, one used contiguous strip lesions (contiguous lesions produced between two probes) at the lateral dorsal foraminal aperture plus monopolar lesioning (lesion produced by one probe) at the L5 dorsal ramus, one targeted the sacral lateral branch using sensory-stimulation guiding, and one targeted the L4-L5 primary dorsal rami and S1-S3 lateral branches. The study by Vallejo, which achieved positive short-term but negative long-term results, used pulsed lesioning at the medial branch of $\mathrm{L} 4$, the posterior primary rami of $\mathrm{L} 5$, and the lateral branches of S1 and S2 [2]. The study by Ferrante, which achieved negative short-term and long-term results targeted the SIJ line, which Yin et al. [21] asserts is located laterally from where one can access the lateral branch nerves, since they are embedded within dense ligaments.

\section{Technique}

In addition, some investigators have asserted that some negative results for radiofrequency neurotomy of the SI joint can be attributed to poor procedure technique. As mentioned above, radiofrequency neurotomy lesions dorsal branches of the SIJ, which are extra-articular, not intraarticular. Lesions of these branches can be made in several fashions. Pino et al. studied the correct parameters of radiofrequency ablation and found that practitioners could create contiguous strip lesions when they placed the cannulae $6 \mathrm{~mm}$ or less apart, while they could create unipolar lesions if the cannulae were spaced more than $6 \mathrm{~mm}$ apart. They found that optimal technique to maximize the surface area of the lesion was created when the cannulae were positioned parallel to each other, 4 to $6 \mathrm{~mm}$ apart, and temperature was held at $90^{\circ} \mathrm{C}$ for $120-150 \mathrm{~s}$ [24].

Vallejo used pulsed radiofrequency neurotomy (PRFN) to decrease maximum temperatures and risk of adjacent tissue destruction [22]. In PRFN, needles should be placed perpendicular to the nerve, where the electrical field created is greatest. Non-PRFN, however, uses parallel positioning because the lesion is created at the tissues surrounding the needle [22]. Vallejo et al. asserts that Ferrante's negative results may have been due to perpendicular positioning of a non-PRFN technique [22]. Indeed, because radiofrequency neurotomy creates relatively small lesions, the positioning of the electrode to the target neural tissue is critically important [21].

Finally, some investigators have targeted particular branches of particular rami, while Burnham and Yasui in a less technically complicated method used a series of bipolar strip lesions adjacent to the lateral dorsal foraminal aperture plus conventional monopolar lesioning at the L5 dorsal ramus, with positive results.

\section{Efficacy}

Percutaneous radiofrequency neurotomy has been found in the literature to have utility in relieving SIJ pain. Hansen 
et al. [2] recently completed a systematic review of SIJ interventions and found limited evidence for the usefulness of radiofrequency neurotomy in managing SIJ pain. The investigators found five retrospective reports of radiofrequency ablation of the SIJ that met key Agency for Healthcare Research and Quality (AHRQ), Quality Assessment for Diagnostic Accuracy Studies (QUADAS), and Cochrane Review Group criteria for studies, including a requirement for greater than 3 months of follow-up. Three of the studies showed positive short-term (less than 3 months) and long-term (greater than or equal to 3 months) pain relief, while one study by Vallejo et al. showed positive short-term and negative long-term relief, and another study by Ferrante et al. showed negative shortterm and negative long-term relief.

Ferrante studied records of 33 patients with SIJS who were diagnosed by a single anesthetic block and found that only $36 \%$ of patients had greater than a $50 \%$ decrease in visual analog pain scores at 6 months [25]. The results were judged to be negative by Hansen's systematic review, and Yin et al. [21] asserts that some of the patients in Ferrante's report may have had other causes of their back pain, since only a single diagnostic block was used.

Vallejo et al. found in their prospective case series of 22 patients with SIJS diagnosed by comparative anesthetic blocks who had failed physical therapy and SIJ injections, 16 patients $(73 \%)$ had pain relief from radiofrequency neurotomy. Of these 16 patients, 4 patients had relief for 6-9 weeks, 5 patients had relief for 10-16 weeks, and 7 patients had relief for 17-32 weeks [22]. Pain relief was measured by visual analog score (VAS) and was considered to be adequate if greater than 50\% reduction in VAS was achieved. The technique used the treatment of radiofrequency neurotomy was PRFN of the medial branch of $\mathrm{L} 4$, posterior primary rami of L5, and lateral branches of S1 and S2. PRFN consisted of radiofrequency current at short bursts as opposed to continuous current, in order to allow cooling of tissues between pulses and less tissue destruction and therefore less pain from the procedure. The authors contend that the temperature of the surrounding tissues does not impact the effectiveness of the procedure and that the mechanism by which radiofrequency neurotomy works is unclear.

Burnham and Yasui evaluated nine patients with SIJ pain confirmed by a local anesthetic block. The investigators treated the patients with radiofrequency neurotomy, consisting of radiofrequency strip lesions adjacent to the lateral dorsal foraminal aperture and conventional monopolar lesioning at the L5 dorsal ramus. Significant reductions of back and leg pain and analgesic intake evaluated by patient questionnaire were found at 1, 3, 6, 9, and 12 months of follow-up [26].
Yin et al. in a retrospective chart review studied 14 patients with SIJS confirmed by double anesthetic blocks and found that $64 \%$ experienced relief of pain after sensorystimulation guided sacral lateral branch radiofrequency neurotomy. Pain relief was defined as greater than $60 \%$ subjective relief and greater than $50 \%$ decrease in visual integer pain score, and the follow-up period was 6 months. The investigators also found that $34 \%$ of the patients reported complete relief [21].

Cohen and Abdi performed radiofrequency neurotomy on nine patients with SIJS confirmed by $50 \%$ pain relief after nerve blocks of the L4-5 primary dorsal rami and S1-S2 lateral branches. The investigators found that $89 \%$ of the nine patients obtained $50 \%$ or greater pain relief after radiofrequency neurotomy of the affected joint at 9 months [27].

\section{Conclusion}

Radiofrequency neurotomy has been shown to have limited evidence in treating SIJS. There are no randomized, controlled trials to evaluate this procedure. Variability in results has been attributed to individual nerve patterns, improper diagnosis, and selection of patients, as well as variability in the procedure itself. Future studies will be needed to focus on the type of radiofrequency neurotomy and positioning of electrodes to better evaluate efficacy.

Open Access This article is distributed under the terms of the Creative Commons Attribution Noncommercial License which permits any noncommercial use, distribution, and reproduction in any medium, provided the original author(s) and source are credited.

\section{References}

1. Leblanc KE. Sacroiliac sprain: an overlooked cause of back pain. Am Fam Physician. 1993;48(8):1388-90.

2. Hansen HC, McKenzie-Brown AM, Cohen SP. Sacroiliac joint interventions: a systematic review. Pain Physician. 2007;10: 165-84.

3. Dunn EJ, Bryan DM, Nugent JT, Robinson RA. Pyogenic infections of the sacroiliac joint. Clin Orthop. 1976;118:113-7.

4. Humphrey SM, Inman RD. Metastatic adenocarcinoma mimicking unilateral sacroiliitis. J Rheumatol. 1995;22:970-2.

5. Fortin JD. Sacroiliac joint dysfunction. A new perspective. J Back Musculoskelet Rehabil. 1993;3:31-43.

6. Slipman CW, Whyte WSII, Chow DW. Sacroiliac joint syndrome. Pain Physician. 2001;4(2):143-52.

7. Fortin JD, et al. Sacroiliac joint innervation and neural structures. Am J Orthop. 1999;28:687-90.

8. Ward S, Jenson M, Royal MA, et al. Fluoroscopy-guided sacroiliac joint injections with phenol ablation for persistent sacroiliitis: a case series. Pain Pract. 2002;2(4):332-5.

9. Dreyfuss P, Dryer S, Grif nJ, Hoffman J, Walsh N. Positive sacroiliac screening tests in asymptomatic adults. Spine. 1994;19:1138-43. 
10. Slipman C, et al. Fluroscopically guided therapeutic sacroilliac joint injections for sacroiliac joint syndrome. Am J Phys Med Rehabil. 2001;80(6):425-32.

11. Laslett M, et al. Diagnosis of sacroilliac joint pain: validity of individual provocation tests and composite tests. Man Ther. 2005;10(3):207-18.

12. Vogler JB III, Brown WH, Helms CA, Genant HK. The normal sacroiliac joint: a CT study of asymptomatic patients. Radiology. 1984;151:433-7.

13. Hanly JG, Mitchell MJ, Barnes DC, MacMillan L. Early recognition of sacroiliitis by magnetic resonance imaging and single photon emission computed tomography [comment]. [Erratum appears in J Rhematol 1997; 24:411-12.] J Rhematol. 1997;21: 2088-95.

14. Lentle BC, Russel AS, Percy JS, Jackson FI. The scintigraphic investigation of sacroiliac disease. J Nucl Med. 1997;18:529-33.

15. Maigne JY, Boulahdour H, Chatellier G. Value of quantitative radionuclide bone scanning in the diagnosis of sacroiliac joint syndrome in 32 patients with low back pain. Eur Spine J. 1998;7:328-31.

16. Battafarano DF, West SG, Rak KM, Fortenbery EJ, Chantelois AE. Comparison of bone scan, computed tomography, and magnetic resonance imaging in the diagnosis of active sacroiliitis. Semin Arthritis Rhem. 1993;23:161-76.

17. Fortin JD, et al. Sacroiliac joint: pain referral maps upon applying a new injection/arthrography technique: part 1, asymptomatic volunteers. Spine. 1994;19:1475-82.
18. Verrills P, Vivian D. Interventions in chronic low back pain. Aust Fam Physician. 2004;33(6):421-6.

19. Dreyfuss P, et al. Sacroiliac joint pain. J Am Acad Orthop Surg. 2004;12:255-65.

20. Forst SL, Wheeler MT, Fortin JD, et al. The sacroiliac joint: anatomy, physiology and clinical significance. Pain Physician. 2006;9:61-8.

21. Yin W, et al. Sensory stimulation-guided sacroiliac joint radiofrequency neurotomy: technique based on neuroanatomy of the dorsal sacral plexus. Spine. 2003;28:2419-25.

22. Vallejo R, et al. Pulsed radiofrequency denervation for the treatment of sacroiliac joint syndrome. Pain Med. 2006;7:429-34.

23. Gevargez A, et al. CT-guided percutaneous radiofrequency denervation of the sacroiliac joint. Eur Radiol. 2002;12:1360-5.

24. Pino CA, et al. Morphologic analysis of bipolar radiofrequency lesions: implications for treatment of the sacroiliac joint. Reg Anesth Pain Med. 2005;30(4):335-8.

25. Ferrante FM, et al. Radiofrequency sacroiliac joint denervation for sacroiliac syndrome. Pain Med. 2004;5(1):26-32.

26. Burnham RS, Yasui Y. An alternate method of radiofrequency neurotomy of the sacroiliac joint: a pilot study of the effect of pain, function, and satisfaction. Reg Anesth Pain Med. 2007; 32:12-9.

27. Cohen SP, Abdi S. Lateral branch blocks as a treatment for sacroiliac joint pain: a pilot study. Reg Anesth Pain Med. 2003;28:113-9. 